\title{
Soziale Verantwortung statt Resignation
}

\section{Auswirkungen konstruktiver Nachrichten auf prosoziale Verhaltensintentionen der Rezipient:innen}

\author{
Julia Steinigeweg
}

Eingegangen: 2. April 2021 / Angenommen: 27. Juli 2021 / Online publiziert: 30. August 2021

(C) Der/die Autor(en) 2021

Zusammenfassung Konstruktiver Journalismus ist eine relativ neue Form des Journalismus mit dem Ziel, die aktuell überwiegend negativ orientierte Medienberichterstattung durch lösungsorientierte Elemente zu ergänzen. Mithilfe von Techniken der positiven Psychologie sollen dem Publikum gesellschaftlich relevante Informationen vermittelt werden, um zu einer mündigen Bevölkerung beizutragen. Dabei nimmt die Stärkung prosozialer Verhaltensintentionen eine zentrale Rolle ein.

Die vorliegende Studie untersucht, inwieweit der konstruktive Journalismus dem eigenen Anspruch einer Förderung proaktiver Verhaltensweisen gerecht wird. In einer experimentellen Befragung wurden prosoziale Selbstwirksamkeitserwartungen, motivationale Faktoren und direkte Verhaltensintentionen untersucht. Die Ergebnisse legen nahe, dass prosoziale Verhaltensintentionen durch die Rezeption konstruktiver Nachrichten gestärkt werden. Ein signifikanter Zusammenhang zwischen der Rezeption konstruktiver Nachrichten und einer gesteigerten Selbstwirksamkeitserwartung ließ sich nicht erkennen.

Schlüsselwörter Konstruktiver Journalismus · Selbstwirksamkeitserwartung · Prosoziales Verhalten $\cdot$ Medienwirkung $\cdot$ Soziale Verantwortung 


\section{Social responsibility instead of resignation}

Effects of constructive journalism on the recipient's prosocial behavioral intentions

Abstract Constructive journalism is a rather new form of journalism aiming at expanding the currently mostly negative media landscape with the help of solutionoriented elements. Although people got used to sensational headlines and catastrophe pictures, they are not immune to (partly unconscious) emotional reactions. Due to increasing resignation and negative feelings towards news, people tend to avoid journalistic reports. The learned helplessness theory indicates that they also reduce the ability to act in a prosocial way. That is exactly the opposite of the original function of journalism: to inform citizens and empower them to participate actively in society and political processes.

Utilizing positive psychology techniques, constructive journalists intend to convey socially relevant information to the audience aiming at contributing to a proactive population. Furthermore, they hope to counteract the journalistic problematics of growing resignation to and avoidance of news.

For that, strengthening prosocial self-efficacy expectations and other motivational factors play a central role. In relation to increased trust in one's own abilities, news consumers become aware of their options for acting. This serves not only psychological well-being, but also significantly promotes social commitment. Overall, by encouraging various recipient's psychological patterns, constructive journalism intends to contribute to an enlightened, mature and prosocial society, in which citizens are aware of their social responsibility and participate in political or charitable processes.

Prosocial behavior is an act that is defined as useful for society. Behavioral intentions are determined by one's own attitude towards this behavior and assumed reactions from confidants. However, unconsciously prosocial behavioral intentions are also determined by expectations of prosocial self-efficacy. Only if someone is convinced that one himself can create the behavior that is useful for society, a real act will follow.

Whether this new journalistic approach complies with its own set claim to contribute to a prosocial minded society, is the central theme of the present work. Accordingly, the superior question of the study, in which way constructive news influence recipient's prosocial behavioral intentions, will be answered in the context of an experimental survey.

Two fictitious online newspaper articles which deal with neighbourhood help during the corona pandemic served as stimuli. One version of the online article reflects constructive reporting including a positive example of how people can participate, and which measures must be taken to reduce the likelihood of spreading the virus. This particular article also explains which alternatives people have in case it is not advisable to help personally, substantiated by an interview with a scientist. Optimistic frames, graphics, direct addressing and a positively connoted picture were used to create this stimulus. The other version of the fictitious newspaper article represents the conventional reporting model. Thereby, a real newspaper article served as a template and only the mentioned place and persons were changed. The latter solely concentrates on the problem that hygiene regulations cannot be adhered to, 
and risk groups are not adequately protected against corona. In addition, the article made use of quotes from critics, a sensational headline, a negatively connoted image and dramatic frames.

In an online survey, the two stimuli were randomly distributed to two groups of test subjects. The newspaper articles were distributed equally among the groups. Consequently, the probability of receiving the constructive newspaper article was $50 \%$.

The results of the two-week survey with a total of 182 valid cases show that constructive news had an influence on the motivation and willingness to produce an actual behavior that is useful for society. As a matter of fact, prosocial self-efficacy expectations were not promoted by them. This is an interesting issue as, based on Bandura's theory of perceived self-efficacy, the conviction of being able to behave in a prosocial manner is the requirement of prosocial behavioral intentions and actual behavior. It should be emphasized that self-efficacy expectations are related to long-lasting personality traits and it is improbable to change such characteristics just because of a one-time reception of a constructive newspaper article. Nevertheless, prosocial behavior is not about convictions or feelings, but rather about actual willingness followed by concrete acts. Thus, the result of the statistical evaluation can be considered successful. Instead of problem-oriented, fearful news reporting that leads to resignation, constructive journalism concentrates on the journalistic core function of enlightenment and looks for solutions to social problems, whereby it strengthens prosocial behavioral intentions.

To conclude, this study points out the tendence that constructive journalism encourages prosocial acts. It could also be proven that news with a solely negative perspective reduce the motivation for prosocial behavioral intentions considerably. Regarding the future, the results should be used as an opportunity to rethink the currently prevailing conviction in Germany of only sensationally negative news causing thoughtfulness and activism.

Keywords Constructive journalism $\cdot$ Self-efficacy $\cdot$ Prosocial behavior $\cdot$ Media effects $\cdot$ Social responsibility

\section{Einleitung}

Seit Jahren werden fortlaufend journalistische Start-Ups gegründet, die als Antwort auf die „Krise des Journalismus“ (Lünenborg 2012, S. 445) gedeutet werden können (vgl. Buschow 2018; Zotta 2020, S. 67) und sinkenden Reichweiten sowie dem wahrgenommenen verminderten Vertrauen in Medien Einhalt gebieten wollen (vgl. Blöbaum 2020, S. 166; Hagen 2015, S. 153). Diese Start-Ups gehen einher mit veränderten professionellen Normen und Ansprüchen, wie qualitativ hochwertiger Journalismus $\mathrm{zu}$ sein hat.

Zweifelsohne sind Digitalisierungsprozesse und damit zusammenhängende frei verfügbare, kostenlose Informationsangebote für den Rückgang der Zeitungsauflagen mitverantwortlich (vgl. Fletcher und Nielsen 2017, S. 1175; Überall 2021). Allerdings verändern sich nicht nur Technologien, sondern auch Gesellschaften stetig, 
und das hat unweigerlich Auswirkungen auf die Berufskultur von Journalist:innen sowie auf Werte, Ziele und Arbeitsweisen des Journalismus im Allgemeinen. So wird auch wachsende Frustration der Öffentlichkeit aufgrund einer vordergründig negativ orientierten Medienberichterstattung (vgl. Kramp und Weichert 2020, S. 85) als Grund dafür angenommen, dass Menschen klassische Medien zunehmend meiden (vgl. Kleemans et al. 2017, S. 2060; Lecheler 2020, S. 297; McIntyre 2015, S. 3). Es fehle eine grundlegende Reflexion über die sich verändernde Rolle des Journalismus im 21. Jahrhundert.

Aus dieser Position heraus argumentiert mitunter der konstruktive Journalismus. Seine Befürworter:innen legen dar, dass fast die Hälfte der Medienvermeider:innen keine Nachrichten mehr rezipieren, weil diese bei ihnen negative Emotionen hervorrufen (vgl. Urner 2019, S. 49). Insbesondere jüngere Menschen scheinen einer wachsenden Resignation zu unterliegen und tagesaktuelle Nachrichten gänzlich zu meiden (vgl. Hermans und Prins 2020, S. 8).

Das Ziel des seit 2015 in journalistischen und kommunikationswissenschaftlichen Debatten diskutierten konstruktiven Journalismus ist es, mithilfe von Techniken der positiven Psychologie sowie der Orientierung an Lösungen ein nutzbringendes und zudem ausgewogenes Bild von Wirklichkeit (als Kompensation zur negativ zentrierten Medienlandschaft) zu vermitteln. Als zentraler Aspekt gilt dabei, soziale Konflikte nicht nur aufzuzeigen, sondern Bürger:innen auch diejenigen Informationen zur Verfügung zu stellen, die sie dazu befähigen, aktiv an der Gesellschaft und (potenziellen) Lösungen teilzuhaben (vgl. Urner 2019, S. 48). Folglich entspricht es dem Rollenbild konstruktiver Journalist:innen, zu einer aufgeklärten, mündigen und prosozialen Gesellschaft beizutragen, in der sich die Bevölkerung ihrer gesellschaftlichen Verantwortung bewusst ist (vgl. Graupmann et al. 2011, S. 117; Kramp und Weichert 2020, S. 16; Pranz und Sauer 2017, S. 112). Rezipient:innen sollen sich nach dem Medienkonsum nicht mehr hilflos und der Welt ausgeliefert fühlen, sondern im Gegenteil ein Gefühl von Selbstwirksamkeit erfahren, das motivationale Kognitionen und die persönliche Handlungsfähigkeit stärkt (vgl. Bandura 1977, S. 193). So sollen das psychische Wohlbefinden, insbesondere jedoch prosoziale Verhaltensintentionen intensiviert werden (vgl. Bandura 1994, S. 2; Bierhoff et al. 2011, S. 82; Curry und Hammonds 2015; Fredrickson 2001, S. 221; Hermans und Gyldensted 2019, S. 539; Kleemans et al. 2017, S. 2060; Levine und Manning 2014, S. 359).

Ob der konstruktive Journalismus diesem Anspruch gerecht wird, ist die zentrale Thematik der vorliegenden Studie. Dementsprechend lautet die übergeordnete Fragestellung, inwieweit konstruktive Nachrichten prosoziale Verhaltensintentionen der Rezipient:innen fördern. Diese Fragestellung soll im Rahmen einer experimentellen Befragung innerhalb Deutschlands beantwortet werden.

\section{Absichten konstruktiver Berichterstattung}

Die Idee, Nachrichten konstruktiv zu gestalten, ist nicht neu. Bei jeder qualitativ hochwertigen Berichterstattung geht es darum, ausgewogen zu berichten und verschiedene Perspektiven einzunehmen, um ein Thema möglichst umfassend zu 
betrachten. Das Konzept eines explizit konstruktiven Journalismus mit spezifischen Merkmalen existiert jedoch erst seit 2015 und ist auf das Buch Constructive News. How to Save the Media and Democracy with Journalism of Tomorrow zurückzuführen, das der dänische Journalist Ulrik Haagerup verfasst hat. Seit der Veröffentlichung des Buches hat dieses Berichterstattungsmuster sowohl in der journalistischen Praxis als auch in der kommunikationswissenschaftlichen Debatte Aufsehen erregt und ist inzwischen Bestandteil vieler fachspezifischer Forschungen, Diskussionen und journalistischer Weiterbildungsmaßnahmen (vgl. Heinrichs 2020; Hooffacker 2020, S. 3; McIntyre und Gyldensted 2018, S. 665).

Im Mittelpunkt dieser teilweise als „Genreinnovation“ (Buschow und Wellbrock 2020, S. 10) bezeichneten journalistischen Richtung steht der Blick in die Zukunft (vgl. Haagerup 2015, S. 144-147). Anstatt nur danach zu fragen, was aktuell der Fall ist, wird nach Lösungen sowie Perspektiven gesucht und auch danach gefragt, wie es weitergehen kann (vgl. Hooffacker 2020, S. 2). Konkret bedeutet dies, dass innerhalb der Berichterstattung selbst bereits existierende Lösungsansätze und Handlungsoptionen aufgezeigt und diskutiert werden, indem die sieben journalistischen W-Fragen Wer? Was? Wo? Wann? Wie? Warum? Woher? Durch die Frage Was nun? ergänzt wird. Dabei ist zu betonen, dass Journalist:innen auf diese Weise weder die Rolle von Aktivist:innen noch von (Politik-)Schaffenden übernehmen (wollen). Vielmehr präsentieren sie mit einer auf Fakten und Argumenten gründenden Haltung Informationen im erklärenden Kontext (vgl. Pranz und Sauer 2017, S. 112; Urner 2019, S. 141). Dementsprechend wird auch über Themen berichtet, bei denen es noch keine bzw. keine zufriedenstellenden Lösungen gibt. Anders formuliert: Konstruktive Journalist:innen sehen es als ihre Aufgabe an, zu einer aufgeklärten und mündigen Bevölkerung beizutragen (vgl. Kramp und Weichert 2020, S. 78), indem sie durch verschiedene Perspektiven eine erweiterte Sicht auf gesellschaftliche Konflikte und vorhandene Lösungen aufzeigen, wenngleich sie selbst keine eigenen Lösungen entwerfen. Dennoch ist der Aspekt nicht zu vernachlässigen, dass sich eine geäußerte journalistische Haltung in der Praxis nicht immer von einer aktivistischen Einstellung unterscheiden lässt. Dies ist auch der größte, immer wieder aufkommende Kritikpunkt in der Debatte um das vergleichsweise neue Berichterstattungsmuster (vgl. Beiler und Krüger 2018, S. 181).

Konstruktiver Journalismus beruht auf den Techniken der positiven Psychologie, die sich wissenschaftlich mit einem lebenswerten Leben auseinandersetzt (vgl. Seligman et al. 2005; Seligman und Csikszentmihalyi 2000). Auf Grundlage des von Seligman (2010) erstellten PERMA-Modells wurden Fragen für die journalistische Praxis formuliert, die darauf abzielen, den Blick auf die positiven Aspekte eines Ereignisses einzubeziehen und so der Resignation auf Nutzer:innenseite vorzubeugen (vgl. Beiler und Krüger 2018, S. 177): Wer engagiert sich? Wer hat ein Problem gelöst? Wer hat dabei geholfen? Was wurde erreicht und wie konnte es erreicht werden? Welche Beispiele für gelingende Kooperationen gibt es? Gibt es eine Inspiration für andere? (vgl. McIntyre und Gyldensted 2018, S. 28-29). Dabei ist konstruktiver Journalismus keineswegs mit positivem Journalismus gleichzusetzen. Positiver Journalismus berichtet über rein positive Ereignisse oder Geschichten und hat das primäre Ziel, positive Emotionen in Rezipient:innen hervorzurufen (vgl. Tomoff 2017, S. 226-227). Diese Form der journalistischen Berichterstattung kann zwar positive 
Auswirkungen auf die Weltanschauung und Einstellungen der Rezipient:innen haben (vgl. Fredrickson 2001, S. 223; Lecheler 2020, S. 290), wird dem Kriterium der Ausgewogenheit aber nicht gerecht. Auch wenn sowohl das konstruktive als auch das positive Berichterstattungsmuster positive Emotionen hervorrufen und zu Handlungen ermächtigen möchten (vgl. McIntyre 2015, S. 81), unterscheiden sie sich grundlegend in ihrer Nachrichtenauswahl (vgl. Fink 2015, S. 10; Jackson 2016, S. 10). Konstruktive Journalist:innen fühlen sich dazu verpflichtet, möglichst umfassend zu berichten und die Kernfunktionen des Journalismus aufrechtzuerhalten, weshalb für sie eine kritische Berichterstattung aller Themen mit gesellschaftlicher Relevanz obligatorisch ist. Sie klären weiterhin über Missstände auf, lassen jedoch dabei positive Entwicklungen nicht außer Acht. Während positiver Journalismus und besonders an negativen Ereignissen orientierter Sensationsjournalismus die beiden Extrema der Berichterstattung darstellen und Wahrnehmungsverzerrungen fördern, macht es sich der konstruktive Journalismus zur Aufgabe, möglichst alle Seiten und Aspekte eines Themas einzubeziehen.

Ein weiteres, oftmals mit dem konstruktiven Journalismus verwechseltes Berichterstattungsmuster ist der Solutions Journalism. Wie der Name bereits verrät, bietet der lösungsorientierte Journalismus potenzielle Antworten auf gesellschaftlich relevante Probleme. Gegenstand seiner Berichterstattung sind institutionelle oder soziale Konflikte sowie der Umgang damit. Dabei geht es nicht nur darum aufzuzeigen, was funktioniert, sondern auch wie und warum eine Lösung zu funktionieren scheint und welche möglichen Nachteile sie mitbringt bzw. was verbesserungswürdig ist (vgl. Curry und Hammonds 2015, S. 5-6). Lösungsorientierter Journalismus weist dementsprechend viele Parallelen zum konstruktiven Journalismus auf, und in der englischen Fachliteratur werden die beiden Begriffe oft synonym verwendet (vgl. Curry und Hammonds 2015; Gyldensted 2011; McIntyre 2019; McIntyre und Gyldensted 2018). Jedoch setzen sie an unterschiedlichen Ausgangspunkten an: Lösungsorientierter Journalismus argumentiert vor allem methodisch und rückt die handwerkliche Dimension auf Inhalts- und Rechercheebene in den Mittelpunkt. Anhänger:innen des konstruktiven Journalismus haben vordergründig das normative Ziel, einen Mentalitätswandel in Redaktionen hervorzurufen (vgl. Kramp und Weichert 2020, S. 33-34). In der Praxis sind beide Berichterstattungsmuster jedoch nicht trennscharf voneinander zu untercheiden. Der lösungsorientierte Journalismus kann somit als Teilbereich des konstruktiven Journalismus verstanden werden (vgl. Hooffacker 2020, S. 4), da er methodische Instruktionen für konstruktive Arbeitsweisen geben will.

Konstruktiver Journalismus ergänzt das klassische Selbstverständnis eines ausgewogenen Qualitätsjournalismus. Konstruktive Journalist:innen sprechen tagesaktueller oder investigativer Berichterstattung keineswegs die Relevanz ab, sondern betonen die Notwendigkeit neuer Techniken und Arbeitsweisen, die das Gesamtbild des Journalismus erweitern und rehabilitieren (vgl. Kramp und Weichert 2020, S. 52; Mast et al. 2019, S. 500). Die bisherige Forschung belegt die Tendenz, dass insbesondere die Altersgruppe der Millennials, die zwischen den frühen 1980erJahren bis zu den späten 1990er-Jahren geboren wurde, konstruktive Nachrichten wünscht (vgl. Bundesverband Deutscher Zeitungsverleger 2017; Hooffacker 2020, S. 4; Kramp und Weichert 2020, S. 16). Anstatt zu resignieren, würden sie nicht mehr über soziopolitisch relevante Meldungen hinwegsehen, sondern wieder Freu- 
de an der Nachrichtenrezeption empfinden und mit ihrem sozialen Umfeld teilen (vgl. Curry und Hammonds 2015, S. 4; Hermans und Prins 2020, S. 13-14; McIntyre 2020, S. 50; Meier 2018, S. 774). Allerdings ist an dieser Stelle ausdrücklich von einer Tendenz die Rede, da aufgrund des jungen Untersuchungsgegenstandes Wirkmechanismen noch wenig untersucht wurden und noch keine Langzeitstudien vorliegen.

\section{Auswirkungen auf prosoziale Verhaltensintentionen}

Der konstruktive Journalismus setzt sich explizit für mehr soziale Verantwortung und prosoziale Verhaltensweisen ein (vgl. Beiler und Krüger 2018, S. 180; Kleemans et al. 2017, S. 2060; Kramp und Weichert 2020, S. 79). Als prosoziales Verhalten gilt eine Handlung, die die Gesellschaft als nützlich für andere Menschen definiert. Diese Handlung kann sowohl aus egoistischen als auch aus altruistischen bzw. uneigennützigen Gründen erfolgen. Nicht mit eingeschlossen sind jedoch Verhaltensweisen, die durch berufliche Verpflichtungen motiviert sind, wie es bspw. bei Krankenpfleger:innen der Fall ist (vgl. Bierhoff 2002, S. 189; Levine und Manning 2014, S. 360-362). Welches Verhalten als nützlich für andere Menschen gilt, ist kulturell unterschiedlich, wird aber auch durch eigene - wiederum von der Gesellschaft geprägte - Werte beeinflusst. Dementsprechend deckt prosoziales Verhalten eine Vielfalt von Phänomenen ab, die im Alltag sowie in Ausnahmesituationen (bspw. bei Naturkatastrophen oder Pandemien) auftreten können (vgl. Bierhoff et al. 2011, S. 62; Penner et al. 2005, S. 4). Auch journalistische Medien können als Vermittler kollektiver Überzeugungen dienen, somit können sie die als gesellschaftlich nützlich erachteten Verhaltensnormen maßgeblich mitprägen.

Soziopsychologisch betrachtet bedarf es einiger Faktoren, um sich tatsächlich prosozial verhalten zu können. In erster Linie muss eine Verhaltensabsicht bzw. intention vorliegen. Nach Icek Ajzens Theory of Planned Behavior (1985) ${ }^{1}$ ist die Verhaltensintention durch die Einstellung zum Verhalten sowie durch die subjektive Norm determiniert. Als subjektive Norm gelten dabei die vermuteten Erwartungen und Konsequenzen, die das soziale Umfeld wegen des Verhaltens zeigen könnte (vgl. Ajzen 1985, S. 13). Steht das Individuum dem Verhalten grundsätzlich positiv gegenüber und überwiegen die wahrgenommenen Belohnungen des sozialen Umfelds die wahrgenommenen Kosten bzw. den Aufwand für die Ausführung des Verhaltens, kommt es nach Ajzens Theorie zur Verhaltensintention. Dies allerdings nur, wenn der Mensch das Gefühl hat, er kann die Situation mit seinem Verhalten beeinflussen bzw. innerhalb eines bestimmten Rahmens kontrollieren (vgl. Ajzen 1985, S. 24). Mit anderen Worten: Er benötigt ein gewisses Maß an Selbstwirksamkeitserwartung 2 .

„Perceived self-efficacy“ (Bandura 1977, S. 193), also die Selbstwirksamkeitserwartung, ist definiert als die subjektive Gewissheit des Menschen, durch eige-

\footnotetext{
${ }^{1}$ Die mit Martin Fishbein ursprünglich 1980 verfasste Theory of Reasoned Action wurde von Ajzen fünf Jahre später zur Theory of Planned Behavior modifiziert bzw. erweitert.

2 In der deutschen Fachliteratur werden die Begriffe Selbstwirksamkeitserwartung und Selbstwirksamkeit häufig synonym verwendet.
} 
ne Fähigkeiten eine gewünschte Handlung ausführen bzw. Anforderungssituationen bewältigen zu können (vgl. Bandura 2012, S. 10; Schwarzer und Jerusalem 2002, S. 29). Entwickelt wird sie maßgeblich durch die jahrelange, subjektive Interpretation von Rückmeldungen des sozialen Umfelds, weshalb sie zu den langanhaltenden Persönlichkeitsmerkmalen gehört (vgl. Bandura 1994, S. 9-14). Da zwischen allgemeiner und bereichsspezifischer Selbstwirksamkeitserwartung differenziert wird, kann sich eine Person in einem bestimmten Lebensbereich wirksam fühlen, wohingegen sie in einem anderen das Gefühl von Hilflosigkeit erfährt. Zwar können Selbstwirksamkeitserwartungen durch Umwelteinflüsse und individuelle Einstellungsänderungen gefördert werden, dies bedarf jedoch zum einen eines langwierigen (vgl. Bandura 2012, S. 12), intrinsisch motivierten Prozesses sowie zum anderen genügend extrinsische Zuspruchs (vgl. Bandura 1994, S. 3).

Prosoziale Selbstwirksamkeitserwartung ist demnach die Überzeugung, ein für die Gesellschaft nützliches Verhalten hervorbringen zu können. Allein diese Überzeugung macht Personen offener für neue Erfahrungen, kontaktfreudiger und sozialer im Umgang mit anderen (vgl. Bandura 1994, S. 11). Personen, die im Vergleich zu anderen eine höhere prosoziale Selbstwirksamkeitserwartung aufweisen, zeigen zugleich ein höheres Maß an prosozialen Verhaltensintentionen und Verhaltensweisen (vgl. Tabernero und Hernández 2011, S. 669-670).

Konstruktiver Journalismus zeigt innerhalb von Konfliktsituationen Handlungsoptionen auf und möchte Rezipient:innen damit das Gefühl vermitteln, etwas bewirken zu können. Somit wäre es zumindest theoretisch möglich, dass dieses Berichterstattungsmuster zu einer prosozialen Selbstwirksamkeitserwartung beiträgt. Da bisherige Studien diese eigens gesetzte Rollenanforderung tendenziell belegen (vgl. Hermans und Gyldensted 2019, S. 548; Hermans und Prins 2020, S. 13; McIntyre 2015, S. 80), werden folgende Zusammenhänge vermutet und empirisch überprüft:

H1: Personen, die konstruktive Nachrichten rezipieren, sind eher der Überzeugung, ein für die Gesellschaft nützliches Verhalten hervorbringen zu können, als jene, die konventionelle Nachrichten rezipieren (Verstärkerhypothese).

H1a: Personen, die geringe Selbstwirksamkeitserwartungen mitbringen, weisen nach der Rezeption konstruktiver Nachrichten ein ähnlich hohes Maß an prosozialer Selbstwirksamkeitserwartung auf, wie jene, die durchschnittliche Selbstwirksamkeitserwartungen mitbringen und konventionelle Nachrichten rezipieren (Kompensationshypothese).

Für die Ausübung prosozialer Verhaltensweisen sind neben der Überzeugung, ein für die Gesellschaft nützliches Handeln hervorbringen zu können, noch andere spezifische Selbstwirksamkeitserwartungen notwendig. Wer die Überzeugung besitzt, theoretisch einen gesellschaftlichen Mehrwert liefern zu können, jedoch keine Zeit oder Ausdauer dafür aufbringen kann oder möchte, wird nicht prosozial agieren. Somit beschäftigt sich die zweite Hypothese mit Selbstwirksamkeitserwartungen, die individuelle Ressourcen betreffen: 
H2: Personen, die konstruktive Nachrichten rezipieren, sind eher der Überzeugung, die nötigen Ressourcen aufbringen zu können, um ein für die Gesellschaft nützliches Verhalten hervorzubringen, als jene, die konventionelle Nachrichten rezipieren (Verstärkerhypothese).

H2a: Personen, die geringe Selbstwirksamkeitserwartungen mitbringen, sind nach der Rezeption konstruktiver Nachrichten genauso davon überzeugt, die nötigen Ressourcen aufbringen zu können, um sich für die Gesellschaft nützlich zu verhalten, wie jene, die durchschnittliche Selbstwirksamkeitserwartungen mitbringen und konventionelle Nachrichten rezipieren (Kompensationshypothese).

Ist ein Individuum der Überzeugung, ein für die Gesellschaft nützliches Verhalten hervorbringen und weiterhin die dafür notwendigen Ressourcen aufbringen zu können, erfährt es eine subjektiv wahrgenommene Verhaltenskontrolle (vgl. Ajzen 1985, S. 36; Schwarzer und Jerusalem 2002, S. 40) bzw. das Gefühl, die Situation beeinflussen zu können (vgl. Bandura 1977, S. 211-212). Wie bereits erwähnt, muss das Individuum für eine tatsächliche Verhaltensabsicht jedoch auch glauben, dass das ausgeführte Verhalten sozial erwünscht ist, damit sich die individuell aufzuwendenden Ressourcen (Zeit, Ausdauer, körperliche Anstrengung, Empathie, Kenntnisse sowie Fähigkeiten) durch soziale Belohnungen wie Anerkennung oder das Zugehörigkeitsgefühl zu einer Gruppe ausgleichen. Indem konstruktive Beiträge sozial engagiertes Verhalten und prosoziale Normen hervorheben und damit Empathie und Anteilnahme erzeugen möchten, könnte auch diese Kosten/Nutzen-Abwägung durch konstruktive Nachrichten positiv beeinflusst werden. Entsprechend lautet Hypothese H3:

H3: Personen, die konstruktive Nachrichten rezipieren, sind eher der Überzeugung, dass sich der Aufwand, ein für die Gesellschaft nützliches Verhalten hervorzubringen, lohnt, als jene, die konventionelle Nachrichten rezipieren (Verstärkerhypothese).

Gehen Personen davon aus, dass ein prosoziales Verhalten lohnenswert ist, sind sie auch eher motiviert, derartige prosoziale Handlungen auszuüben. Folglich wird prognostiziert:

H3a: Personen, die konstruktive Nachrichten rezipieren, fühlen sich eher motiviert, ein für die Gesellschaft nützliches Verhalten hervorzubringen, als jene, die konventionelle Nachrichten rezipieren (Verstärkerhypothese).

H3b: Personen, die geringe Selbstwirksamkeitserwartungen mitbringen, sind nach der Rezeption konstruktiver Nachrichten genauso motiviert, ein für die Gesellschaft nützliches Verhalten hervorzubringen, wie jene, die durchschnittliche Selbstwirksamkeitserwartungen mitbringen und konventionelle Nachrichten rezipieren (Kompensationshypothese). 


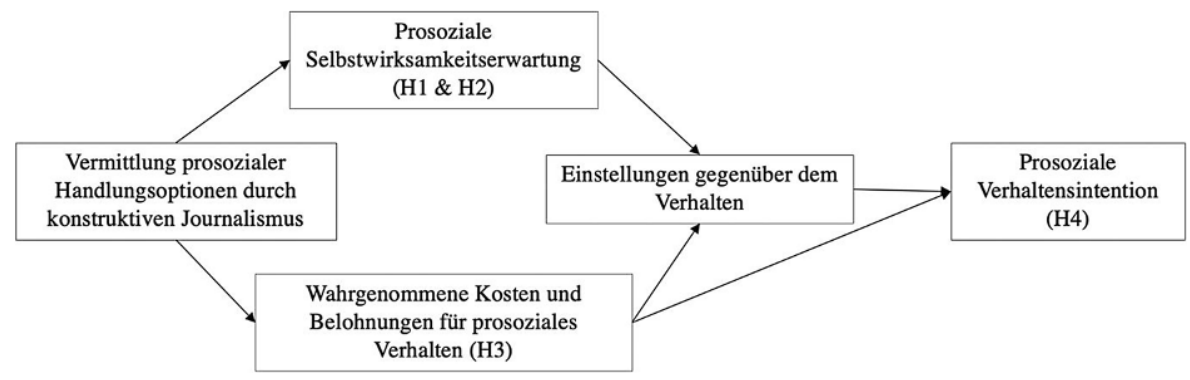

Abb. 1 Faktoren prosozialer Verhaltensintentionen mit Einbezug der Funktion journalistischer Berichterstattung

Wenn spezifische Selbstwirksamkeitserwartungen vorhanden sind und die subjektive Abwägung wahrgenommener Kosten und sozialer Belohnungen zugunsten prosozialer Verhaltensweisen ausfallen, stellt sich eine prosoziale Verhaltensintention ein (vgl. Ajzen 1985, S. 33). Zwar spielen dann immer noch situative Faktoren für die tatsächliche Ausführung prosozialer Verhaltensweisen eine Rolle, die Wahrscheinlichkeit einer solchen Handlung ist jedoch stark erhöht (vgl. Abb. 1). Dementsprechend wird angenommen:

H4: Personen, die konstruktive Nachrichten rezipieren, sind eher dazu bereit, ein für die Gesellschaft nützliches Verhalten hervorzubringen, als jene, die konventionelle Nachrichten rezipieren (Verstärkerhypothese).

H4a: Personen, die geringe Selbstwirksamkeitserwartungen mitbringen, sind nach der Rezeption konstruktiver Nachrichten im gleichen Maße dazu bereit, ein für die Gesellschaft nützliches Verhalten hervorzubringen, wie jene, die durchschnittliche Selbstwirksamkeitserwartungen mitbringen und konventionelle Nachrichten rezipieren (Kompensationshypothese).

\section{Methode}

Eine quantitative experimentelle Befragung diente dazu, die Hypothesen zu prüfen. Stimuli waren dabei zwei fiktive Zeitungsartikel, die im Rahmen einer OnlineBefragung per Zufallsverfahren auf die Proband:innen verteilt wurden. Der eine Zeitungsartikel stellt ein konventionelles, der andere ein konstruktives Berichterstattungsmuster dar. Dabei erfolgte die Aufteilung der zwei Versionen des Zeitungsartikels auf die Versuchspersonen gleichmäßig, sodass sich am Ende der Befragung zwei Vergleichsgruppen mit derselben Personenanzahl ergaben.

Operationalisierung Die beiden Zeitungsartikel thematisieren die Nachbarschaftshilfe während der Corona-Pandemie. Notwendige Informationen für die Erstellung der Artikel wurden aus Beiträgen der Süddeutschen Zeitung und der Zeit 
sowie Ratgeber-Seiten der Caritas und des ADAC (vgl. Caritas Deutschland 2020; Frisse 2020; Thielitz 2020; Trummer 2020) entnommen und modifiziert.

Da sich ein konstruktives Berichterstattungsmuster an der Suche nach Lösungen orientiert, zeigt die erste Version des Stimulus anhand eines Positivbeispiels auf, welche Möglichkeiten es gibt, sich zu beteiligen. Durch die Überschrift „Wie auch Du helfen kannst. Ein Netzwerkforscher erklärt, wie Du Deine Nachbarn während Corona sicher unterstützen kannst - ohne Dein Sozialleben zu opfern" werden Rezipient:innen direkt angesprochen. Unmittelbar am Anfang des Artikels wird zunächst die Relevanz und der gesellschaftliche Konflikt des Themas aufgezeigt, indem eine Beschreibung beispielhafter Einschränkungen im Alltag stattfindet: „Die CoronaPandemie stellt viele vor große Herausforderungen. Ältere Menschen sollten soziale Kontakte meiden - also auch den Supermarkt. Eltern müssen arbeiten und gleichzeitig ihre Kinder betreuen. Und wer unter Quarantäne gestellt wird, darf gar nicht mehr vor die Tür. Auch Einrichtungen wie die Tafel oder Sozialläden bekommen immer weniger Lebensmittel und andere Gebrauchsgegenstände aus den Supermärkten und Drogerien gespendet." Mit diesem Einstieg soll verhindert werden, dass der Stimulus eher dem Berichterstattungsmuster eines positiven statt eines konstruktiven Journalismus gleicht. Anschließend nennt der Artikel mögliche Hilfeleistungen in der Nachbarschaft und verweist immer wieder auf Maßnahmen, die getroffen werden müssen, damit die Wahrscheinlichkeit einer weiteren Ausbreitung des Virus vermindert werden kann.

Weiterhin erläutert ein Wissenschaftler, welche Personengruppen nicht aktiv helfen sollten, da sie Vorerkrankungen oder viel Kontakt zu anderen Menschen haben und sich Infektionsketten durch ihre direkte Hilfe ausweiten könnten. Auch für diese Gruppen gibt der Experte Lösungsvorschläge an die Hand, wie sie durch Spenden oder Telefondienste trotz Kontaktvermeidung Hilfe leisten können. Eine Grafik erklärt anschaulich, wie viele Menschen sich schon bei der Nachbarschaftshilfe engagieren, indem sie für Bedürftige einkaufen, Lebensmittel spenden, den Hund von Nachbar:innen ausführen, Kinder betreuen, sich bei der Telefonseelsorge engagieren und an Nachbarschaftsinitiativen spenden. Optimistische Frames wie Herausforderung statt Problem oder dürfen statt müssen sowie die direkte Ansprache werden für die Vermittlung eines prosozialen Selbstwirksamkeitsgefühls verwendet. Als weitere Unterstützung dient ein positiv konnotiertes Bild, das eine Instagram-Influencerin in einer Wohnsiedlung geschossen hat und auf dem bunte Masken an einem Gartenzaun zu sehen sind.

Die zweite Version des fiktiven Zeitungsartikels stellt das konventionelle Berichterstattungsmuster dar. Als Vorlage diente hierbei ein realer Online-Artikel der Süddeutschen Zeitung (vgl. Trummer 2020), der bis auf Standortinformationen und Eigennamen vollständig übernommen wurde. Anstelle von Positivbeispielen und Lösungsansätzen konzentriert sich diese Version allein auf die Problematik, dass Hygienevorschriften nicht eingehalten und Risikogruppen nicht ausreichend vor Corona geschützt werden können. Durch die Überschrift „Angst vor der Tafel“ und ein Bild, das einen Senior allein auf der Straße mit Einkaufstasche und Krückstock zeigt, ist unmittelbar ein sensationeller, negativ konnotierter Bezugsrahmen erkennbar. Unterstützend wirken dramatisierende Frames wie stören, fürchten oder Probleme sowie 
Zitate von Kritiker:innen, die eine Tafel während der Corona-Pandemie als „sehr bedenklich“ erachten.

Stichprobe Die Stichprobenziehung erfolgte durch eine Gelegenheitsauswahl. Anschließend wurde der Fragebogen per Schneeballverfahren weitergeleitet. Zwar ist eine randomisierte Stichprobe einer Gelegenheitsstichprobe aufgrund der Repräsentativität immer vorzuziehen, da die Studie jedoch im Rahmen einer Masterarbeit entwickelt wurde und nicht durch externe oder hochschulinterne Mittel mitfinanziert werden konnte, trug das spätere Schneeballverfahren zumindest zu einem gewissen Grad an Heterogenität des Samples bei. Je länger die Befragung andauerte, desto mehr ältere Personen nahmen an der Befragung teil: In der ersten Hälfte des Befragungszeitraums betrug das Durchschnittsalter der Befragten 27 Jahre, in der zweiten Hälfte waren die Teilnehmer:innen durchschnittlich 37 Jahre alt. Daraus ist zu schließen, dass Versuchspersonen der Ausgangsstichprobe den Fragebogen an ältere Personen weitergeleitet haben, die ihn zu einem späteren Zeitpunkt ausgefüllt haben. Eine Varianz beim Bildungsgrad konnte durch das ergänzte Schneeballverfahren allerdings nicht erzielt werden, und auch die Geschlechterverteilung war in der ersten und zweiten Hälfte des Befragungszeitraums sehr ähnlich (diese war allerdings schon vor dem ergänzten Schneeballverfahren ausgewogen).

Das Durchschnittsalter der Versuchspersonen beträgt 32 Jahre, wobei zu betonen ist, dass knapp $45 \%$ der Befragten zwischen 22 und 25 Jahre alt sind. Somit handelt es sich keineswegs um ein demografisch repräsentatives Sample, da jedoch insbesondere diese Altersgruppe konstruktive Nachrichten fordert (vgl. Bundesverband Deutscher Zeitungsverleger 2017; Kramp und Weichert 2020, S. 16) und einige vom neuen Berichterstattungsmuster erwarteten Effekte an der Generation der Millennials verifiziert wurden (vgl. Hermans und Gyldensted 2019, S. 543; Hermans und Prins 2020, S. 14; McIntyre 2020, S. 50; Meier 2018, S. 774), eignet sich das in dieser Studie erfasste Sample durchaus für eine Analyse der Auswirkungen konstruktiver Nachrichten auf prosoziale Selbstwirksamkeitserwartungen. Ein weiterer Vorteil des Samples ist, dass in dieser Altersspanne vor allem die Förderung jener bereichsspezifischen Selbstwirksamkeitserwartungen eine Rolle spielt, die sich auf soziale Verantwortlichkeit beziehen (vgl. Bandura 1994, S. 12).

Insgesamt nahmen 55\% weibliche und $43 \%$ männliche Personen an der Befragung teil, eine Versuchsperson ist divers. Knapp zwei Prozent gaben ihr Geschlecht nicht an. Alle befragten Personen haben ihren Wohnort in Deutschland, wobei die meisten in Münster (47\%), Lippstadt (7\%) oder Köln (7\%) wohnen. Keine der Versuchspersonen hat gar keinen oder einen Hauptschulabschluss. Als höchsten Abschluss gaben drei Prozent einen Realschulabschluss, 34\% Abitur und 60\% aller Befragten einen Hochschulabschluss an (2\% kreuzten die Auswahlkategorie Sonstiges an). Damit handelt es sich nicht nur um ein sehr junges, sondern auch um ein überdurchschnittlich gebildetes Sample.

Durchführung Die Befragung lief über die Online-Befragungsplattform SoSci Survey. Nach dem Pretest am 5. Juni 2020 fand die eigentliche Erhebung zwischen dem 9. und dem 24. Juni 2020 statt. 
Tab. 1 Abhängige Variablen

\begin{tabular}{ll}
\hline Hypothese & Abhängige Variablen \\
\hline H1 \& H1a & Prosoziale Selbstwirksamkeitserwartung \\
H2 \& H2a & Selbstwirksamkeitserwartungen hinsichtlich persönlicher Ressourcen, dazu zählen \\
& 1. Kenntnisse \\
& 2. Fähigkeiten \\
& 3. Zeit \\
& 4. Ausdauer \\
& 5. Körperliche Kraft \\
& 6. Empathie \\
& Erwartete Belohnungen, dazu zählen \\
H3a & 2. Soziale Belohnungen (Anerkennung) \\
& 3. Materialistische Belohnungen (Gegenleistung in Form von Wertgegenständen oder \\
& Geld) \\
H3b \& H3c & Motivation \\
H4a \& H4b & Bereitschaft, Hilfeverhalten zu leisten
\end{tabular}

In dem Fragebogen wurden vor dem Einsatz des Stimulus soziodemographische Daten und die generelle Selbstwirksamkeitserwartung abgefragt. Es ist zu vermuten, dass die bereits vorhandene allgemeine Selbstwirksamkeitserwartung erheblichen Einfluss auf die prosoziale Selbstwirksamkeitserwartung hat. Somit fungiert die vorher abgefragte allgemeine Selbstwirksamkeitserwartung (niedrig, durchschnittlich, hoch) als unabhängige Kontrollvariable, um potenziell messbare Effekte valide auf die Rezeption verschiedener Berichterstattungsmuster rückführen bzw. Deckeneffekte ausschließen zu können. Für die Messung der Kovariaten fand die Generalized Self-Efficacy Scale von Schwarzer und Jerusalem (1995) Anwendung, die vier Jahre später von Schwarzer (1999) ins Deutsche übersetzt wurde. Anhand einer fünfstufigen Skala konnten die Testpersonen ankreuzen, ob die Aussagen zu ihren allgemeinen Selbstwirksamkeitserwartungen vollkommen auf sie zutreffen (5), eher zutreffen (4), eher nicht zutreffen (2), überhaupt nicht zutreffen (1) oder ob sie sich unschlüssig sind (3).

Nach dem Einsatz des Stimulus, der in dem Experiment als unabhängige Variable fungierte, wurden explizite Fragen zu den abhängigen Variablen gestellt (vgl. Tab. 1). Auch hier erfolgte die Abfrage - abgesehen von den für $\mathrm{H} 4$ und $\mathrm{H} 4 \mathrm{a}$ relevanten Variablen, bei denen eine Mehrfachauswahl an (vorgegebenen) Aussagen getroffen werden konnte - anhand einer fünfstufigen Ankreuz-Skala.

Die Ankreuzmöglichkeiten der abhängigen Variable prosoziale Selbstwirksamkeitserwartung bezogen sich auf die Überzeugungen der Testpersonen hinsichtlich ihrer prosozialen Verhaltensweisen in der Corona-Pandemie, wie ,Ich bin der Überzeugung, dass ich Menschen, die meine Hilfe während Corona benötigen, unterstützen kann“ oder „Ich bin der Überzeugung, dass meine Hilfe nützlich ist, auch wenn ich nicht vor Ort bin“. Bei den spezifischen Selbstwirksamkeitserwartungen waren die Aussagen ähnlich aufgebaut. So lautete die Aussage zur abhängigen Variablen Kenntnisse bspw. „Ich bin der Überzeugung, dass ich die nötigen Kenntnisse besitze, um helfen zu können“. Auch die Variablen erwartete Belohnungen und Motivation wurden so operationalisiert, dass Proband:innen ihre Überzeugungen einschätzen 
sollten, ob sich ein Hilfeverhalten während Corona lohnt. Für Hypothese 4 und $4 \mathrm{a}$ war die Frage zu beantworten, inwieweit Testpersonen bereit wären, ihr soziales Umfeld während Corona zu unterstützen. Dabei wurden in Bezug auf die prosoziale Verhaltensintention Hilfeleistungen aufgezählt (z. B.: „Ich wäre bereit, für Bedürftige einzukaufen oder Botendienste zu erledigen“ oder „Ich wäre bereit, in Not geratene Freunde finanziell zu unterstützen“). In einem „Sonstiges“-Feld konnten Proband:innen weitere Hilfeleistungen notieren.

Um den Fragebogen abschließen zu können, mussten die Testpersonen alle Fragen beantworten, ausgenommen die der Soziodemographie. Insgesamt füllten 247 Teilnehmer:innen den Fragebogen aus, von denen 212 alle Fragen beantworteten und als gültige Fälle für die Auswertung herangezogen wurden.

Auswertungsstrategie Um den Einfluss der unabhängigen Variable (Stimulus in Form eines modifizierten Zeitungsartikels) auf die metrisch skalierten abhängigen Variablen zu messen, fanden Berechnungen von einfaktoriellen Varianzanalysen statt. Um die Hypothesen zu messen, welche die Kontrollvariable allgemeine Selbstwirksamkeitserwartung mit einbezogen, wurden einfaktorielle Kovarianzanalysen durchgeführt ${ }^{3}$.

Vor der eigentlichen Analyse sind Indizes für die in mehreren Aussagen operationalisierten abhängigen Variablen prosoziale Selbstwirksamkeitserwartung (Cronbachs $\alpha=0,92$ ), spezifische Selbstwirksamkeitserwartung (Cronbachs $\alpha=0,78$ ), erwartete Belohnungen (Cronbachs $\alpha=0,83$ ) und prosoziale Verhaltensintention (Cronbachs $\alpha=0,69)$ sowie für die Kontrollvariable allgemeine Selbstwirksamkeitserwartung (Cronbachs $\alpha=0,97$ ) gebildet worden. Die Indexbildung erfolgte durch eine Mittelwertberechnung der durchschnittlichen Skalenwertangaben der einzelnen Aussagen. Nur die abhängige Variable Motivation wurde anhand einer Aussage gemessen (,Ich bin der Überzeugung, dass ich genügend Motivation besitze, um anderen zu helfen“).

Die Kontrollvariable allgemeine Selbstwirksamkeitserwartung wurde zudem nach gering, durchschnittlich und hoch gruppiert. Aufgrund der Verwendung einer fünfstufigen Abfrageskala bot es sich an, Testpersonen, welche die zehn Aussagen zu ihrer allgemeinen Selbstwirksamkeitserwartung mit einem Index-Wert von 1 (trifft überhaupt nicht $\mathrm{zu}$ ) bis 2,3 (trifft eher nicht $\mathrm{zu}$ ) beantworteten, als Personen mit niedriger allgemeiner Selbstwirksamkeitserwartung einzustufen. Versuchspersonen, bei denen der Mittelwert für die Variable allgemeine Selbstwirksamkeitserwartung zwischen 2,4 und 3,6 Skalenpunkten lag, wurden als Personen mit durchschnittlichen allgemeinen Selbstwirksamkeitserwartungen angesehen, und Personen, bei denen der Mittelwert 3,7 oder höher betrug, wurden als Personen mit hoher allgemeiner Selbstwirksamkeitserwartung betrachtet. Insgesamt wiesen 38 Versuchsper-

\footnotetext{
3 Die Voraussetzungen zur Durchführung der Varianz- und Kovarianzanalysen wurden weitestgehend erfüllt, mit Ausnahme der Voraussetzung auf Normalverteilung bei der abhängigen Variable Motivation (diese ist linkssteil) sowie der Varianzhomogenität innerhalb der Gruppen. Da beide Analyseverfahren jedoch verhältnismäßig robust gegen Ausreißer sind und alle anderen Voraussetzungen erfüllt wurden, erfolgte die weitere Berechnung mit gleichbleibender Stichprobe.
} 
sonen eine niedrige, 97 Personen eine durchschnittliche und 77 Personen eine hohe allgemeine Selbstwirksamkeitserwartung auf.

\section{Ergebnisse und Diskussion}

Nach Hypothese 1 sind Testpersonen, die konstruktive Nachrichten rezipieren, eher der Überzeugung, ein für die Gesellschaft nützliches Verhalten hervorbringen zu können, als jene, die konventionelle Nachrichten rezipieren. Die ANOVA zeigte allerdings keinen signifikanten Mittelwertunterschied zwischen dem Einfluss des konstruktiven $(M=3,95 ; S D=0,37)$ und dem Einfluss des konventionellen Zeitungsartikels $(M=3,78 ; S D=0,39)$ auf die prosoziale Selbstwirksamkeitserwartung $(p=0,76)$, weshalb Hypothese 1 verworfen wird. Nach der ANCOVA mit Einbezug der Kovariate allgemeine Selbstwirksamkeitserwartung blieb der Einfluss nicht signifikant $(p=0,45)$, sodass auch Hypothese $1 a$ als falsifiziert gelten muss. Der Einfluss der allgemeinen Selbstwirksamkeitserwartung auf die prosoziale Selbstwirksamkeitserwartung wies allerdings ein signifikantes Ergebnis auf $(p<0,001)$. Die Kontrollvariable hat ihren Zweck dementsprechend an dieser Stelle erfüllt: Vermutlich hat die bereits vorhandene allgemeine Selbstwirksamkeitserwartung einen großen Einfluss auf das Empfinden von prosozialen Selbstwirksamkeitserwartungen, unabhängig davon, welche Art von Nachricht rezipiert wird.

Bei $H 2$ und $H 2 a$, die sich mit den spezifischen Selbstwirksamkeitserwartungen bei individuellen Ressourcen befassen, die prosoziale Verhaltensweisen begünstigen, wurde hinsichtlich der Auswertung genauso verfahren wie bei Hl und Hla. Auch hier ergab sich nach der ANOVA für Hypothese 2 kein signifikanter Mittelwertunterschied zwischen dem Einfluss des konstruktiven $(M=3,72 ; S D=0,46)$ und dem des konventionellen Zeitungsartikels $(M=3,63 ; S D=0,52)$ auf die abhängige Variable $(p=0,92)$. Ebenso verhielt es sich bei der ANCOVA für Hypothese $2 a(p=0,82)$. Somit gelten beide Hypothesen als falsifiziert: Personen, die konstruktive Nachrichten rezipieren sind nicht eher davon überzeugt, die nötigen Ressourcen aufbringen zu können, um ein für die Gesellschaft nützliches Verhalten hervorzubringen, als jene, die konventionelle Nachrichten rezipieren - unabhängig davon, welches Maß an allgemeiner Selbstwirksamkeitserwartung sie besitzen.

Da es sich bei dem kognitiven Konstrukt der Selbstwirksamkeitserwartung um ein Persönlichkeitsmerkmal handelt, das sich nur durch konstante, langfristige extrinsische und intrinsische Einflussfaktoren ändern kann (vgl. Bandura 1994, S. 9), sind diese Auswertungsergebnisse nicht überraschend. Um eine Änderung von Persönlichkeitseigenschaften hervorzurufen, bedarf es mehr als der einmaligen Rezeption einer konstruktiven Nachricht. Da die Mittelwerte der abhängigen Variable allerdings bei Rezipient:innen der konstruktiven Nachricht immer höher waren, wäre es sinnvoll, $H 1$ und $H 2$ in zukünftigen Längsschnittstudien mit einem breiter angelegten Studiendesign abermals zu prüfen.

Gemäß Hypothese 3 wären Personen, die konstruktive Nachrichten rezipieren, eher der Überzeugung, dass sich der Aufwand, ein für die Gesellschaft nützliches Verhalten hervorzubringen, lohnt, als jene, die konventionelle Nachrichten rezipieren. Diese Hypothese wird jedoch nach dem nicht signifikanten Mittelwertunter- 
Abb. 2 Anzahl der angegebenen prosozialen Verhaltensintentionen (H4)

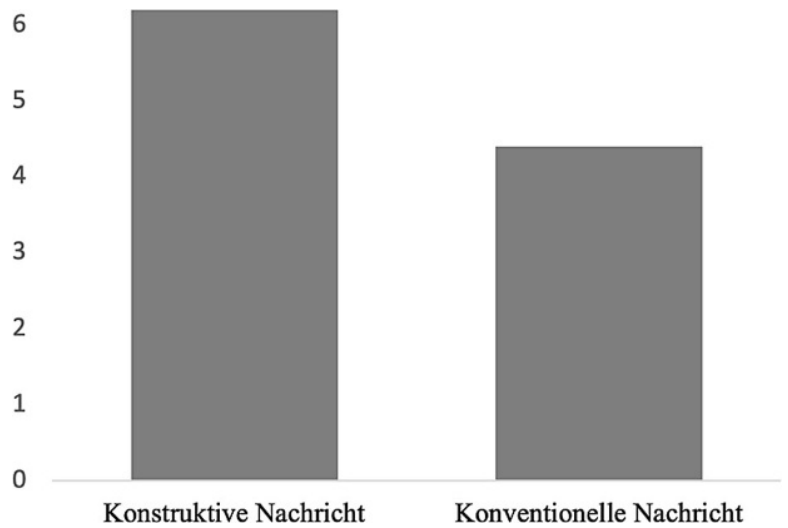

schied zwischen dem Einfluss des konstruktiven $(M=3,36 ; S D=0,42)$ und dem des konventionellen Artikels $(M=3,42 ; S D=0,45)$ auf die abhängige Variable erwartete Belohnungen verworfen $(p=0,90)$. Eine mögliche Erklärung für das nicht signifikante Ergebnis wäre auch in diesem Fall, dass sich die erwarteten Belohnungen für eine prosoziale Handlung aus langjährigen Erfahrungen ergeben, die grundlegender wirken, als dass sie durch die einmalige Lektüre eines konstruktiven Artikels verändert werden könnten.

Bezüglich Hypothese $3 a$ und $3 b$ verhielt es sich ähnlich wie bei den ersten beiden Hypothesen. Personen, welche die konstruktive Nachricht rezipierten $(M=3,74$; $S D=1,30$ ), fühlten sich - gemessen an den Mittelwerten - eher motiviert, ein für die Gesellschaft nützliches Verhalten hervorzubringen, als jene, welche die konventionelle Nachricht rezipierten $(M=3,62 ; S D=1,04)$. Jedoch war auch dieser Mittelwertunterschied nicht signifikant $(p=0,22)$, ebenso wenig wie der Mittelwertunterschied mit Berücksichtigung der Kovariate $(p=0,23)$. Dementsprechend gelten Hypothese $3 a$ und $3 b$ als falsifiziert. Jedoch wies die ANCOVA einen signifikanten Einfluss der allgemeinen Selbstwirksamkeitserwartung auf die Motivation auf $(p<0,001)$, somit sind Menschen mit einer höheren allgemeinen Selbstwirksamkeitserwartung eher motiviert, sich prosozial zu engagieren.

Die letzten beiden Hypothesen beziehen sich auf die tatsächliche Bereitschaft, ein für die Gesellschaft nützliches Verhalten hervorzubringen. Damit sollen keine Überzeugungen oder Empfindungen, sondern Handlungsintentionen messbar gemacht werden.

Nach Hypothese 4 wären Personen, die konstruktive Nachrichten rezipieren, eher dazu bereit, ein für die Gesellschaft nützliches Verhalten hervorzubringen, als jene, die konventionelle Nachrichten rezipieren. Die ANOVA ergab hier erstmals einen signifikanten Mittelwertunterschied $(p=0,003)$, und die prosoziale Verhaltensintention der Testpersonen, welche die konstruktive Nachricht rezipierten $(M=1,62$; $S D=0,18)$, war höher als die von Testpersonen, welche die konventionelle Nachricht zu lesen bekamen $(M=1,44 ; S D=0,17)$, womit sich Hypothese 4 bestätigt.

Die Bereitschaft, während der Corona-Pandemie Hilfe zu leisten, ist anhand einer Mehrfachauswahl operationalisiert worden. Die Versuchspersonen konnten aus mehreren Aussagen, die ihre Verhaltensabsichten widerspiegeln sollten (bspw. „Ich 


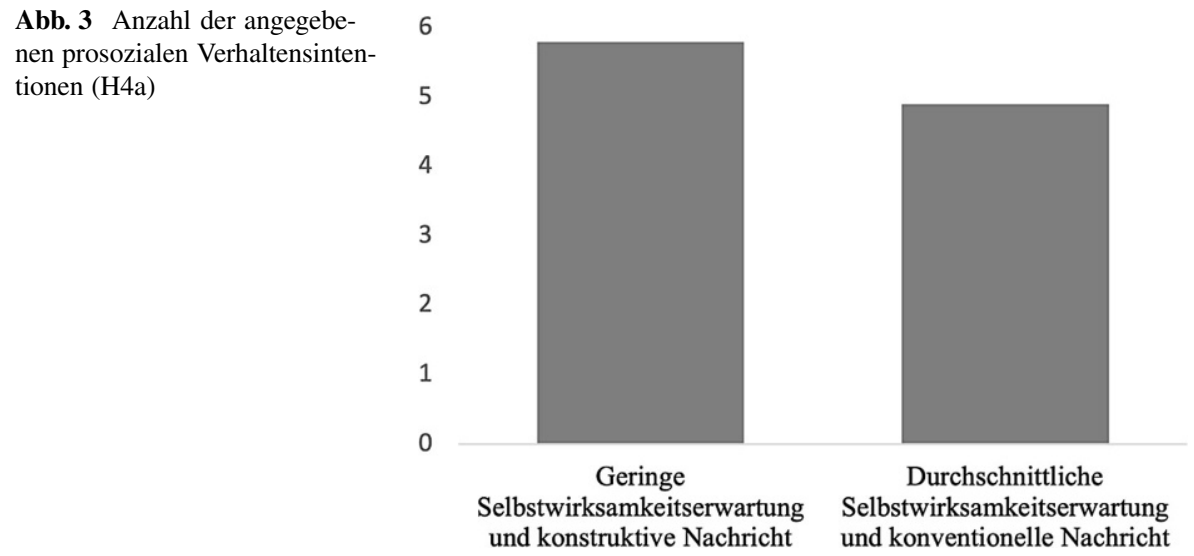

wäre bereit, Geld an eine Initiative zu spenden"), beliebig viele auswählen. Bei insgesamt zehn Auswahloptionen bedeutet der Mittelwertunterschied von 0,18, dass Testpersonen, die den konstruktiven Zeitungsartikel zu lesen bekamen, bereit waren, knapp zwei prosoziale Verhaltensweisen mehr auszuführen, als jene, die den konventionellen Zeitungsartikel gelesen haben (vgl. Abb. 2). Somit wird das signifikante Ergebnis der ANOVA als inhaltlich aussagekräftig gedeutet: Konstruktive Nachrichten haben einen positiven Einfluss auf prosoziale Verhaltensintentionen.

Die ANCOVA für Hypothese 4 a war ebenfalls signifikant $(p=0,004)$. Dementsprechend ist der Einfluss des Stimulus auf die prosoziale Verhaltensintention unabhängig von der allgemeinen Selbstwirksamkeitserwartung der Testpersonen. Proband:innen, die geringe Selbstwirksamkeitserwartungen mitbringen und die konstruktive Nachricht rezipiert haben, sind eher dazu bereit, sich sozial zu engagieren $(M=1,58 ; S D=0,20)$, als jene, die durchschnittliche Selbstwirksamkeitserwartungen mitbringen und die konventionelle Nachricht zu lesen bekamen $(M=1,49$; $S D=0,12)$. Damit wurde ein stärkerer Effekt des Stimulus gemessen, als in Hypothese 4a ursprünglich vermutet (vgl. Abb. 3).

Interessant ist, dass die allgemeine Selbstwirksamkeitserwartung bei $\mathrm{H} 4$ und $H 4 a$ keinen signifikanten Einfluss auf die prosoziale Verhaltensintention besaß $(p=0,14)$, was gegen die Theorie der Selbstwirksamkeitserwartung nach Bandura (1977) spricht, die von einem direkten Einfluss der Selbstwirksamkeitserwartung auf Verhaltensintentionen ausgeht.

Insgesamt werden durch die statistische Auswertung die Teilhypothesen $H 4$ und $H 4 a$ bestätigt, die restlichen Hypothesen gelten als falsifiziert. Die Ergebnisse zeigen, dass konstruktive Nachrichten einen positiven Einfluss auf die Bereitschaft der Rezipient:innen besitzen, ein für die Gesellschaft nützliches Verhalten hervorzubringen. Interessanterweise werden prosoziale Verhaltensintentionen entgegen Albert Banduras Theorie der Selbstwirksamkeitserwartung (1977) in dieser Studie nicht durch die allgemeine Selbstwirksamkeitserwartung beeinflusst. Die allgemeine Selbstwirksamkeitserwartung hat jedoch verständlicherweise einen Einfluss auf prosoziale und andere spezifische Selbstwirksamkeitserwartungen. Auch die Motivation, sich prosozial zu verhalten, wird von ihr mitmoduliert. 


\section{Limitationen}

Bei diesen Ergebnissen sind einige methodische Limitationen zu berücksichtigen. Zunächst ist die geringe Anzahl an Versuchspersonen (212 gültige Fälle) zu nennen. Diese ist auf die Bedingungen einer Studie innerhalb einer Masterarbeit zurückzuführen.

Beim Stimulus fand kein „Manipulation Check“ statt, dementsprechend muss sich die statistische Signifikanz des Einflusses auf Verhaltensintentionen nicht zwangsläufig auf die Unterschiede zwischen konstruktivem und konventionellem Berichterstattungsmuster beziehen. Da der für die Studie herangezogene Zeitungsartikel durch Frames, Darstellungsweisen und inhaltliche Aspekte manipuliert wurde, könnte der positive Einfluss auch auf Einzelindikatoren zurückzuführen sein und nicht unbedingt auf die gesamte Aufbereitungsform. Allerdings kommt es beim konstruktiven Journalismus auf das Zusammenspiel der Einzelindikatoren an, die innerhalb der journalistischen Aufbereitung alle mitbedacht werden müssen. Insofern kann argumentiert werden, dass es keine große Rolle spielt, ob nur ein oder mehrere Charakteristika der konstruktiven Aufbereitungspraktik zu prosozialen Verhaltensintentionen führen.

Wie bereits erwähnt zählen Selbstwirksamkeitserwartungen laut Bandura (1977) zu den Persönlichkeitsmerkmalen, die relativ stabil sind und sich nur durch das Zusammenspiel von langanhaltenden Umweltfaktoren und individuellen Kognitionsprozessen verändern lassen. Somit ist es insgesamt nicht sehr wahrscheinlich, durch die einmalige Rezeption eines konstruktiven Zeitungsartikels einen Einfluss auf Selbstwirksamkeitserwartungen messen zu können. Es bestand aufgrund des Forschungsdesigns von vornherein nur die Möglichkeit, gewisse Tendenzen für die Auswirkungen konstruktiver Nachrichten auf prosoziale Selbstwirksamkeitserwartungen aufzuzeigen.

Der Fragebogen bestand insbesondere bei den operationalisierten Variablen allgemeine und prosoziale Selbstwirksamkeitserwartung aus relativ vielen, ähnlich aufgebauten Aussagen, weshalb es möglich ist, dass Testpersonen im Laufe der Befragung zu müde wurden, um alle Aussagen exakt zu lesen und zu beantworten.

Zudem besteht in experimentellen Befragungen immer das Risiko einer Ergebnisverzerrung durch das Bedürfnis der Versuchspersonen nach sozialer Erwünschtheit, wodurch eine höhere Bereitschaft prosozialer Verhaltensweisen angegeben worden sein könnte, als real vorhanden sind. Dennoch zeigen die Ergebnisse einen signifikanten Unterschied zwischen Rezipient:innen, die den konstruktiven Zeitungsartikel gelesen haben, und jenen, die den konventionellen Zeitungsartikel gelesen haben. Setzt man ein in den beiden Gruppen annähernd gleiches Bedürfnis nach sozialer Erwünschtheit voraus, ist dieser Kritikpunkt für das Ergebnis der vorliegenden Arbeit folglich nicht weiter relevant.

\section{Fazit und Ausblick}

Der konstruktive Journalismus entwickelt sich zu einer Zeit, in der soziokulturelle, technologische und wirtschaftliche Trends zu weitreichenden Veränderungen in 
der Gesellschaft führen. Unser heutiges Wissen und die Menge an Informationen steigt durch die Technisierung und Digitalisierung erheblich (vgl. Ortner et al. 2016, S. 157). In der „,network society“ (Hermans und Drok 2018, S. 689) stehen Informationen kostenlos für alle und zu jeder Uhrzeit frei zur Verfügung, wodurch die Bevölkerung prinzipiell nicht mehr auf journalistische Berichterstattungen angewiesen ist. Folglich ist es nötig, die Kernfunktionen des Journalismus neu zu definieren. Konsequenterweise sind technologische und wirtschaftliche Faktoren in diese Überlegungen mit einzubeziehen; dennoch reichen sie nicht aus, um den professionellen Journalismus vor dem Verlust der sozialen Relevanz durch verschiedenste Akteur:innen im Internet (bspw. Influencer:innen oder Unternehmen, die „Native Advertising“ betreiben) zu bewahren. Aus journalistischer Perspektive ist es notwendig, alte Ziele, Werte und Normen an die sich rasant ändernden Umweltfaktoren anzupassen. Dabei ist es zu einer zentralen Fragestellung der Journalismusforschung geworden, wie mit gesellschaftlich relevanten Themen im öffentlichen Diskurs so umgegangen werden kann, dass Rezipient:innen Handlungsmöglichkeiten erhalten, anstatt mit Resignation, Skepsis und Misstrauen zu reagieren.

Es steht außer Frage, dass das Berichten von gesellschaftlichen Konflikten eine der wesentlichen Aufgaben des Journalismus darstellt. Gleichzeitig ist die berühmte Aussage „Only bad news is good news“ zum Credo der gesamten Branche geworden (vgl. Urner 2017). Im konstruktiven Journalismus geht es nicht darum, soziale Missstände schön zu reden, sondern im Gegenteil die Bevölkerung durch die nötigen Informationen darin zu bestärken, gegen die Missstände vorgehen zu können. Er erkennt an, dass Journalist:innen eine aktive Rolle bei der Konstruktion medienvermittelter Realität spielen, und versteht seine Rezipient:innen als sozial kompetente Bürger:innen, welche die Fähigkeit besitzen, eigenverantwortlich zu handeln und journalistische Konstruktionen funktional zu interpretieren (vgl. Hermans und Drok 2018, S. 686).

Nach den Ergebnissen der vorliegenden Studie fördern konstruktive Nachrichten die Absicht, sich prosozial zu engagieren. Auch Personen, die sich im Allgemeinen als wenig handlungsfähig einschätzen, zeigten eine gesteigerte prosoziale Verhaltensintention nach der Rezeption einer konstruktiven Nachricht. Es erfolgte zwar keine Untersuchung hinsichtlich tatsächlicher Verhaltensweisen, jedoch führt eine prosoziale Verhaltensintention nach Ajzens Theory of Planned Behavior (1985) zu einer realen Handlung, sollten situative Faktoren stimmen.

Prosoziale Persönlichkeiten mit der überdauernden Tendenz, an die Rechte und das Wohl anderer zu denken, Empathie und Anteilnahme zu empfinden und so zu handeln, dass es anderen nützt, übernehmen soziale Verantwortung (vgl. Montada 2001; Schwartz 1977). Somit zeigt das Ergebnis der Studie die Tendenz auf, dass eine langfristige Rezeption konstruktiver Nachrichten zu einer sozial verantwortlich handelnden Gesellschaft führen könnte, wenn schon moderate kurzfristige Änderungen gemessen werden können. Dieses Fazit ist jedoch im Kontext des limitierten Studiendesigns zu betrachten. Um allgemeingültige Aussagen treffen zu können, bedarf es einer Untersuchung mit breiterer Stichprobenbasis und mit Personen, die konstant konstruktive Nachrichten konsumieren.

Die in den letzten sechs Jahren zu dieser Thematik vorgelegten Querschnittsstudien haben konträre Ergebnisse hervorbracht. Während Curry und Hammonds (2015) 
eine signifikante Steigerung von Selbstwirksamkeitserwartungen und Verhaltensintentionen durch die Rezeption konstruktiver Nachrichten gemessen haben, konnte McIntyre (2015) - entgegen den Ergebnissen der vorliegenden Studie - nur eine gesteigerte Selbstwirksamkeitserwartung, aber keine gesteigerte Verhaltensintention messen. Diese inkohärenten Resultate unterstützen die Notwendigkeit zukünftiger Längsschnittstudien, und zwar in Form von Feldexperimenten unter realen Bedingungen. Weiterhin möglich wären Befragungen von Personen, die bereits über einen längeren Zeitraum hinweg konstruktive Nachrichten rezipieren. Ersteres würde viele (finanzielle und zeitliche) Ressourcen in Anspruch nehmen. Letzteres könnte zu dem Problem führen, dass Personen, die regelmäßig konstruktive Nachrichten konsumieren, eine spezifische Soziodemographie und so von vornherein eine höhere prosoziale Selbstwirksamkeitserwartung aufweisen, die durch andere Faktoren als die Rezeption konstruktiver Nachrichten zustande kommen kann. Insofern wären künftig sich ergänzende Untersuchungen mit unterschiedlichen Studiendesigns, Operationalisierungen und Fragestellungen von Vorteil. Zusätzlich ließe sich an die hier vorgestellte Studie anknüpfen, indem untersucht wird, ob konstruktiver Journalismus nicht nur kurzweilige prosoziale Verhaltensweisen und nachbarschaftliches Engagement, sondern generell prosoziale Wertvorstellungen und damit einhergehende langanhaltende Ziele, wie bspw. den Eintritt in eine ehrenamtliche Organisation oder den Berufswunsch, beeinflussen.

Ferner wäre es wichtig zu untersuchen, ob sich theoretisch formulierte Handlungsempfehlungen in der Praxis umsetzen lassen und wie sie im Rahmen des journalistischen Berufsalltags bewertet werden. Außerdem sollten sich Kommunikationswissenschaftler:innen neben der Analyse konstruktiver Texte auch mit anderen Elementen der Nachrichtenberichterstattung beschäftigen, bspw. mit Schlagzeilen, (Bewegt-)Bildern und Audioformaten. Insbesondere die Analyse von schnelllebigen Nachrichten in sozialen Netzwerken sowie Push-Benachrichtigungen verdienten hinsichtlich ihrer steigenden Relevanz mehr Aufmerksamkeit.

Funding Open Access funding enabled and organized by Projekt DEAL.

Open Access Dieser Artikel wird unter der Creative Commons Namensnennung 4.0 International Lizenz veröffentlicht, welche die Nutzung, Vervielfältigung, Bearbeitung, Verbreitung und Wiedergabe in jeglichem Medium und Format erlaubt, sofern Sie den/die ursprünglichen Autor(en) und die Quelle ordnungsgemäß nennen, einen Link zur Creative Commons Lizenz beifügen und angeben, ob Änderungen vorgenommen wurden.

Die in diesem Artikel enthaltenen Bilder und sonstiges Drittmaterial unterliegen ebenfalls der genannten Creative Commons Lizenz, sofern sich aus der Abbildungslegende nichts anderes ergibt. Sofern das betreffende Material nicht unter der genannten Creative Commons Lizenz steht und die betreffende Handlung nicht nach gesetzlichen Vorschriften erlaubt ist, ist für die oben aufgeführten Weiterverwendungen des Materials die Einwilligung des jeweiligen Rechteinhabers einzuholen.

Weitere Details zur Lizenz entnehmen Sie bitte der Lizenzinformation auf http://creativecommons.org/ licenses/by/4.0/deed.de. 


\section{Literatur}

Ajzen, I. (1985). From intentions to actions: a theory of planned behavior. In J. Kuhl \& J. Beckmann (Hrsg.), Action-control: From cognition to behaviour (S. 11-39). Berlin: Springer.

Ajzen, I., \& Fishbein, M. (1980). Understanding attitudes and predicting social behavior. Englewood Cliffs: Prentice Hall.

Bandura, A. (1977). Self-efficacy: toward a unifying theory of behavioral change. Psychological Review, 84, 191-215.

Bandura, A. (1994). Self-efficacy. In S. Ramachaudran (Hrsg.), Encyclopedia of human behavior (S. 71-81). New York: Academic Press.

Bandura, A. (2012). On the functional properties of perceived self-efficacy revisited. Journal of Management, 38, 9-44.

Beiler, M., \& Krüger, U. (2018). Mehr Mehrwert durch Konstruktiven Journalismus? In N. Gonser (Hrsg.), Der öffentliche (Mehr-)Wert von Medien: Public Value aus Publikumssicht (S. 167-191). Wiesbaden: Springer.

Bierhoff, H.-W. (2002). Prosocial behaviour. Hove: Psychology Press.

Bierhoff, H.-W., Frey, D., \& Rohmann, E. (2011). Positive Psychologie: Glück, Prosoziales Verhalten, Verzeihen, Solidarität, Bindung, Freundschaft. In H.-W. Bierhoff \& D. Frey (Hrsg.), Sozialpsychologie Interaktion und Gruppe (S. 81-105). Göttingen: Hogrefe.

Blöbaum, B. (2020). (Medien-)Vertrauen in einer digitalisierten Welt. In S. Ückert, H. Sürgit \& G. Diesel (Hrsg.), Digitalisierung als Erfolgsfaktor für das Sozial- und Wohlfahrtswesen (S. 165-174). BadenBaden: Nomos.

Bundesverband Deutscher Zeitungsverleger (2017). Millennials ticken anders. http://www.bdzv.de/ nachrichten-und-service/presse/pressemitteilun- gen/artikel/detail/millennials_ticken_anders/. Zugegriffen: 4. Mai 2020.

Buschow, C. (2018). Die Neuordnung des Journalismus. Eine Studie zur Gründung neuer Medienorganisationen. Wiesbaden: Springer VS.

Buschow, C., \& Wellbrock, C.-M. (2020). Die Innovationslandschaft des Journalismus in Deutschland. Düsseldorf: Landesanstalt für Medien NRW.

Caritas Deutschland (2020). Wie kann ich jetzt helfen? Nachbarschaftshilfe und Ehrenamt in der CoronaKrise. https://www.caritas.de/magazin/schwerpunkt/corona/wie-kann-ich-jetzt-helfen-nachbarscha fts. Zugegriffen: 28. Mai 2020.

Curry, A.L., \& Hammonds, K.H. (2015). The power of solutions journalism. engaging news project. https://engagingnewsproject.org/research/solutions-journalism/. Zugegriffen: 4. Mai 2020.

Fink, C. (2015). Positiver Journalismus - einführende Gedanken. In Deutscher Fachjournalisten-Verband (Hrsg.), Positiver Journalismus (S. 7-17). Konstanz: UVK.

Fletcher, R., \& Nielsen, R. K. (2017). Paying for Online news. A comparative analysis of six countries. Digital Journalism, 5, 1173-1191.

Fredrickson, B.L. (2001). The role of positive emotions in positive psychology. American Psychologist, $56,218-226$.

Frisse, J. (2020). Nachbarschaftshilfe: Nicht einfach draufloshelfen. https://www.zeit.de/zeit-magazin/ leben/2020-03/nachbarschaftshilfe-coronavirus-infektionskette-solidaritaet-netzwerkforschung (Erstellt: 19. März 2020). Zugegriffen: 29. Mai 2020.

Graupmann, V., Osswald, S., Frey, D., Streicher, B., \& Bierhoff, H.-W. (2011). Positive Psychologie: Zivilcourage, soziale Verantwortung, Fairness, Optimismus, Vertrauen. In D. Frey \& H.-W. Bierhoff (Hrsg.), Sozialpsychologie - Interaktion und Gruppe (S. 107-129). Göttingen: Hogrefe.

Gyldensted, C. (2011). Innovating news journalism through positive psychology. http://repository.upenn. edu/mapp_capstone/20. Zugegriffen: 3. Dez. 2020.

Haagerup, U. (2015). Constructive News. Warum „,bad news“ die Medien zerstören und wie Journalisten mit einem völlig neuen Ansatz wieder Menschen berühren. Salzburg: Oberauer.

Hagen, L. M. (2015). Nachrichtenjournalismus in der Vertrauenskrise. „Lügenpresse“ wissenschaftlich betrachtet: Journalismus zwischen Ressourcenkrise und entfesseltem Publikum. Communicatio Socialis, 48, 152-163.

Heinrichs, E. (2020). Konstruktiver Journalismus. Seminarvortrag „Über Medien informieren“, Grimme Institut, Marl, 15. Okt. 2020.

Hermans, L., \& Drok, N. (2018). Placing constructive journalism in context. Journalism Practice, 12, 679-694. 
Hermans, L., \& Gyldensted, C. (2019). Elements of constructive journalism: characteristics, practical application and audience valuation. Journalism, 20, 535-551.

Hermans, L., \& Prins, T. (2020). Interest matters: the effects of constructive news reporting on Millennials' emotions and engagement. Journalism. https://doi.org/10.1177/1464884920944741.

Hooffacker, G. (2020). Journalistische Praxis: Konstruktiver Journalismus: Wie Medien das Thema Migration für Jugendliche umsetzen können. Wiesbaden: Springer.

Jackson, J. (2016). Publishing the Positive. Exploring the motivations for and the consequences of reading solutions-focused journalism. Masterarbeit. London: University of East London.

Kleemans, M., Schlindwein, L.F., \& Dohmen, R. (2017). Preadolescents' emotional and prosocial responses to negative TV news: investigating the beneficial effects of constructive reporting and Peer discussion. Journal of Youth and Adolescence, 46, 2060-2072.

Kramp, L., \& Weichert, S. (2020). Nachrichten mit Perspektive. Lösungsorientierter und konstruktiver Journalismus in Deutschland. https:/www.otto-brenner-stiftung.de/fileadmin/user_data/stiftung/ 02_Wissenschaftsportal/03_Publikationen/AH101_konstr_Journalismus.pdf. Zugegriffen: 15. Febr. 2021.

Lecheler, S. (2020). The emotional turn in journalism needs to be about audience perceptions: commentary - virtual special issue on the emotional turn. Digital Journalism, 8, 287-291.

Levine, M., \& Manning, R. (2014). Prosoziales Verhalten. In K. Jonas, W. Stroebe \& M. Hewstone (Hrsg.), Sozialpsychologie (S. 357-400). Berlin: Springer.

Lünenborg, M. (2012). Die Krise des Journalismus? Die Zukunft der Journalistik! Ein Diskussionsbeitrag zur Reflexivität und Praxisrelevanz von Wissenschaft. Publizistik, 57, 445-461.

Mast, J., Coesemans, R., \& Temmerman, M. (2019). Constructive journalism: concepts, practices, and discourses. Journalism, 20, 492-503.

McIntyre, K.E. (2015). Constructive journalism: the effects of positvie emotions and solution information in news stories. Doktorarbeit University of North Carolina.

McIntyre, K.E. (2019). Solutions journalism: the effects of including solution information in news stories about social problems. Journalism Practice, 13, 16-34.

McIntyre, K.E. (2020). „Tell me something good“: testing the longitudinal effects of constructive news using the Google assistant. Electronic News, 14, 37-54.

McIntyre, K.E., \& Gyldensted, C. (2018). Positive psychology as a theoretical foundation for constructive journalism. Journalism Practice, 12, 662-678.

Meier, K. (2018). How does the audience respond to constructive journalism? Two experiments with multifaceted results. Journalism Practice, 12, 764-780.

Montada, L. (2001). Denial of responsibility. In A. E. Auhagen \& H.-W. Bierhoff (Hrsg.), Responsibility: the many faces of a social phenomenon (S. 79-92). London: Routledge.

Ortner, H., Pfurtscheller, D., Adolf, M., Rizzolli, M., \& Wiesinger, A. (2016). Datenflut und Informationskanäle. Innsbruck: Innsbruck University Press.

Penner, L. A., Dovidio, J.F., Piliavin, J. A., \& Schroeder, D. A. (2005). Prosocial behavior: multilevel perspectives. Annual Review of Psychology, 56, 365-392.

Pranz, S., \& Sauer, C. (2017). „Konstruktiver Journalismus“- Reflexion über einen Branchentrend, der alte Fragen zum Verhältnis von Pädagogik und Journalismus neu aufwirft. In S. Allmann \& J. TalmonGros (Hrsg.), Kon-Texte: Pädagogische Spurensuche (S. 99-122). Wiesbaden: Springer.

Schwartz, S.H. (1977). Normative influences on altruism. In L. Berkowitz (Hrsg.), Advances in experimental social psychology (S. 221-279). New York: Academic Press.

Schwarzer, R. (1999). Skala zur Allgemeinen Selbstwirksamkeitserwartung. https://www.researchgate.net/ publication/238580838. Zugegriffen: 11. Mai 2020.

Schwarzer, R., \& Jerusalem, M. (1995). Generalized self-efficacy scale. In J. Weinman, S. Wright \& M. Johnston (Hrsg.), Measures in health psychology: a user's portfolio. Causal and control beliefs (S. 35-37). Windsor: NFER-NELSON.

Schwarzer, R., \& Jerusalem, M. (2002). Das Konzept der Selbstwirksamkeit. Zeitschrift für Pädagogik, 44, 28-53.

Seligman, M.E.P. (2010). Flourish: positive psychology and positive interventions. The Tanner Lectures on Human Values, 31, 230-243.

Seligman, M.E.P., \& Csikszentmihalyi, M. (2000). Positive psychology. An introduction. American Psychologist, 55, 5-14.

Seligman, M.E.P., Steen, T. A., Park, N., \& Peterson, C. (2005). Positive psychology progress: empirical validation of interventions. American Psychologist, 60, 410-421.

Tabernero, C., \& Hernández, B. (2011). Self-efficacy and intrinsic motivation guiding environmental behavior. Environment and Behavior, 43, 658-675. 
Thielitz, K. (2020). Nachbarschaftshilfe in Corona-Zeiten: Deutschland solidarisiert sich. https://www. adac.de/news/corona-nachbarschaftshilfe/ (Erstellt: 27. Mai 2020). Zugegriffen: 28. Mai 2020.

Tomoff, M. (2017). Positive Psychologie - Erfolgsgarant oder Schönmalerei? Berlin: Springer.

Trummer, S. (2020). Angst vor der Tafel. https://www.sueddeutsche.de/muenchen/landkreismuenchen/ corona-pandemie-angst-vor-der-tafel-1.4917885 (Erstellt: 25. Mai 2020). Zugegriffen: 28. Mai 2020.

Überall, F. (2021). Chance in der Krise: Bürde oder Chance? Wege aus der Krise. Diskussionsbeitrag, Deutscher Journalistentag des DJV-NRW, Düsseldorf, 20.02..

Urner, M. (2017). Konstruktiver Journalismus. https://perspective-daily.de/konstruktiver_journalismus/ definition. Zugegriffen: 27. März 2020.

Urner, M. (2019). Schluss mit dem täglichen Weltuntergang. Wie wir uns gegen die digitale Vermüllung unserer Gehirne wehren. München: Droemer.

Zotta, F. (2020). Woher nehmen und nicht stehlen?: Der Journalismus und sein Finanzierungsproblem. In J. Schnurr \& A. Mäder (Hrsg.), Wissenschaft und Gesellschaft: Ein vertrauensvoller Dialog (S. 63-84). Berlin: Springer.

Julia Steinigeweg ist Doktorandin am Institut für Kommunikationswissenschaft in Münster. 